\title{
The Volatility Forecasting Power of Financial Network Analysis
}

\author{
Nicolás S. Magner $\mathbb{D}^{1},{ }^{1}$ Jaime F. Lavin $\mathbb{D}^{2},{ }^{2}$ Mauricio A. Valle, ${ }^{1}$ and Nicolás Hardy ${ }^{1}$ \\ ${ }^{1}$ Facultad de Economía y Negocios, Universidad Finis Terrae, Pedro de Valdivia 1509, Providencia, Santiago 7501015, Chile \\ ${ }^{2}$ Escuela de Negocios, Universidad Adolfo Ibáñez, Diagonal Las Torres 2640, Peñalolén, Santiago 7941169, Chile
}

Correspondence should be addressed to Nicolás S. Magner; nmagner@uft.cl

Received 28 May 2020; Accepted 30 July 2020; Published 30 September 2020

Guest Editor: Thiago Christiano Silva

Copyright (c) 2020 Nicolás S. Magner et al. This is an open access article distributed under the Creative Commons Attribution License, which permits unrestricted use, distribution, and reproduction in any medium, provided the original work is properly cited.

\begin{abstract}
This investigation connects two crucial economic and financial fields, financial networks, and forecasting. From the financial network's perspective, it is possible to enhance forecasting tools, since econometrics does not incorporate into standard economic models, second-order effects, nonlinearities, and systemic structural factors. Using daily returns from July 2001 to September 2019, we used minimum spanning tree and planar maximally filtered graph techniques to forecast the stock market realized volatility of 26 countries. We test the predictive power of our core models versus forecasting benchmarks models in and out of the sample. Our results show that the length of the minimum spanning tree is relevant to forecast volatility in European and Asian stock markets, improving forecasting models' performance. As a new contribution, the evidence from this work establishes a road map to deepening the understanding of how financial networks can improve the quality of prediction of financial variables, being the latter, a crucial factor during financial shocks, where uncertainty and volatility skyrocket.
\end{abstract}

\section{Introduction}

In this paper, we used minimum spanning tree length (MSTL) and maximally filtered graph length (PMFGL) methodologies to improve the forecasting of volatility in financial markets. In times of crisis, uncertainty soars, and capital markets evolve quickly and wildly, generating a surge in the volatility of financial assets (a metric for measuring the uncertainty in financial markets is the Chicago Board Options Exchange Volatility Index (CBOE VIX). Since its inception, this index exhibits an average history value of nearly 20 , but during the subprime crisis of 2008-2009 reached its maximum historical level of 89.53. Similarly, nowadays, during the COVID-19 crisis, the index reached a second historic maximum level of 85.47 units), affecting the price behavior, risk management, and asset pricing, also consumption, savings, and investment decisions in the economy, that weakening the economic growth and well-being in the short and long run [1]. Consequently, improving the forecasting of volatility is a priority for its role in portfolio selection, risk management, and derivatives pricing, helping policymakers, institutions, and people to minimize better adverse effects in the postcrisis stage $[2,3]$.
During the past two decades, forecasting models' performance has improved with the incorporation of more data, where the results of the predictions are very reliable on weekly, monthly, and even quarterly horizons [4]. New approaches emerged, from high-frequency models [5] to multivariate $\mathrm{ARCH}$, stochastic volatility models for timevarying return volatilities, and conditional distributions $[6,7]$, and long memory models [8]. Despite these broad advances, volatility models continue to be of very little dimensionality and univariate, which do not manage to incorporate second-order effects and nonlinear relationships typical of complex systems [5].

Complexity is a crucial element for understanding the behavior of financial markets and its reaction to disturbances. An example of a complex system is capital markets characterized by the presence of multiple economic agents interacting simultaneously (complex systems in opposition to linear systems are characterized among other factors, by being nonlinear. In other words, in a nonlinear system, the change in the outputs is nonproportional to the change in the inputs, causing the system to appear chaotic, unpredictable, or even contradictory). The existence of multiple 
entities and various interaction rules (on several degrees and nonlinearities), among other characteristics, generates collective effects that hinder the understanding and modeling of the whole system [9].

One way to improve the understanding of complex systems' behavior is through the use of network methods (they allow modeling the indirect effects in the interconnections of its components or entities. The traditional econometric methods study the direct effects on the relationships of the entities of a system; when using networks, it is feasible to estimate, for example, the distance between two entities or nodes and how likely an indirect effect is between them. These kinds of computations are not feasible using only traditional statistics. This advantage is one of the reasons because network approaches have been used in economics and financial markets). The literature that examines networks in financial markets focuses on the implications of network properties and their relationships with the stability and fragility of financial systems [10, 11]. The literature also explored how the distribution of the links affects the systemic reaction to shocks and how the connectivity of critical nodes or hub nodes could destabilize and even cause the entire network to collapse [12-15]. Likewise, other relevant topics are related to transaction networks of financial assets, portfolio selection, risk management, overlapped portfolios, integration of financial markets, and financial crises [16-22].

Network methodologies apply correlation networks to analyze the synchronization of returns. For the case of interconnectedness risk, planar maximally filtered graph (PMFG) and minimum spanning trees (MST) are used to study the increase in cointegration between financial markets (this phenomenon not only negatively affects investors' possibilities to diversify but is also evidence of an increase in the influence of regional and global economic phenomena on the economies and financial markets that comprise it. For example, Onnela et al. [18] study correlation networks of the S\&P 500, finding the presence of dynamic clusters whose existence is not exclusively due by the industrial sector, but to psychological and economic factors captured in the asset network. They also find that the normalized tree length of the MST (MSTL) is dynamic and reaches minimums during financial crises and that the potential for diversification is related to the evolution of the MSTL of the asset network. During crises, the topology of an MST becomes more starlike and compact, being this network less resilient to shocks and more prone to systemic risk [23]) [24]. We applied minimum spanning tree length (MSTL) and maximally filtered graph length (PMFGL) to measure the synchronization of returns of global stock markets (Granger causality refers to temporary anticipation of an effect (called forecasting power), which may be due to a black box of explanations. We evaluate the predictive power of equity markets via Granger-causality and forecasting regressions, which are useful to assess whether a variable has the predictive ability, not whether it "causes" other variables to change. The latter question can only be answered by using a structural model. However, we can study whether PMFGL and MSTL have predictive ability above and beyond that contained in other variables, such as global demand pressures and interest rates, used as a proxy for the world business cycle, and we undertake such analysis [25]) because both are parsimonious representations of the complex network of interrelationships, and its connections are useful for obtaining direct and indirect information [26]. The ability to represent a dynamic system is a second reason for measuring cointegration with these methodologies. This topic is particularly useful when it is required to represent the phenomenon incorporating a large number of markets under examination [24]. Despite the differences between MSTL and PMFGL, the advantage of reducing the complexity of networks is that it represents nonredundant and the essential connections in a graphical way.

We intend to explore the possibilities of using the MSTL and PMFGL methodologies to improve the forecasting of volatility in financial markets. We hypothesize that there is Granger causality (Granger causality refers to temporary anticipation of an effect (called forecasting power), which may be due to a black box of explanations. We evaluate the predictive power of equity markets via Granger causality and forecasting regressions, which are useful to assess whether a variable has the predictive ability, not whether it "causes" other variables to change. The latter question can only be answered by using a structural model. However, we can study whether PMFGL and MSTL have predictive ability above and beyond that contained in other variables, such as global demand pressures and interest rates, used as a proxy for the world business cycle, and we undertake such analysis [25]) between the global MSTL and global PMFGL and the realized stock market volatility. We think that the global network correlations between the stock markets have relevant information to forecasting realized volatility of stock indices. It is vital to notice that our paper does not study "causality effects," and in other words, we do not study the structural link between the length of MSTL (and PMFGL) and the realized volatility of stock markets.

We contribute to the extant financial network literature in two ways. First, we study an application of the PMFGL and MSTL in the field of forecasting, defining a methodology for testing the predictive power of these two network measures. Second, we connect to relevant fields that, to our best knowledge, have not been linked, network analysis and forecasting. We believe that there is an excellent possibility of contributing financial networks to the field of financial forecasting. Many papers forecast stock market volatility, but none have used financial network metrics that incorporate correlations' asset trees as independent factors [27-29].

To analyze our hypothesis, we test the predictive power of the global MSTL and global PMFGL on the stock market of 26 countries of North America, Latin America, Europe, Asia, and Oceania. For this, we collected daily returns from July 2001 to September 2019 for the main stock indices of these countries and calculated their monthly realized volatility. We then apply finance network methodologies to estimate the PMFGL and MSTL metrics to represent the global correlation structure and to observe it dynamically over time. Finally, we tested the predictive power of such 
network metrics using in-the-sample and out-of-sample tests. Finally, we applied robustness tests to our results.

Our main finding is that MSTL helps to predict the realized volatility of stock markets. Specifically, results indicate that there is Granger causality of the MSTL on the volatility performed in most Asian and European markets. Nevertheless, there is no evidence for the case of North and Latin American markets. This finding would mean that the global correlation network behind the MSTL contains useful information that helps to predict the realized volatility of stock markets. Another relevant result relates to the predictive power of PMFGL. There is evidence that compared to the MSTL, its ability is more limited. One explanation could be that compared with MSTL, this network measure captures more information from the entire asset's correlation network. This would appear to be counterproductive to its volatility predictive power. These results are robust in out-of-sample tests adding benchmark models with six lags, but the effect disappears when we add the variation of the VIX lagged in one month. The previous is preliminary evidence that the MSTL would be an efficient indicator to represent information of the global volatility of the stock markets, with similar predictive power to VIX. However, we think that MSTL has more advantage than the VIX. First, MSTL considers information of all stock markets in comparison with VIX that is elaborated only with the North American stock market. Secondly, MSTL is calculated with realized correlations, and VIX is estimated with expected volatility that is more sensible at market sentiments.

The main conclusion of this paper is that the global correlation connections add useful information that helps to predict the realized volatility in a relevant segment of global stock markets. These results imply that policymakers and practitioners could improve their estimations of future volatility in financial markets and, consequently, improve their forecasting and decision-making regarding asset pricing and risk management. From an economic policy point of view, this work could help policymakers to improve financial stability frameworks and design models that consider the underlying structure of the global network of stock indices. Finally, a possible extension of this work is the development of new methods that deepen the study of the connection between correlations' assets networks and the influence of volatility gauges as the VIX.

This paper is organized as follows. In Section 2, we present the possibilities of expanding the realized volatility forecasting methodologies. In Section 3, we indicate the methodology and the data used in the study. Section 4 shows the results of the in-the-sample and out-of-sample and robustness tests. Section 4 concludes and provides some future research extensions.

\section{Realized Volatility Forecasting Methodologies}

Financial crises have attracted considerable attention from the literature of financial networks. During the financial crisis of 2008-2009, the synchronization of returns, defined as the tendency of stock markets to display significant comovements [30], has a negative impact on the contribution of diversification to risk minimization. This high interconnectedness risk (network centrality measures quantify the interconnectedness risk. The network is built from some measure of dependence between financial assets (e.g., correlations)) phenomenon is an element that becomes a contagion channel for financial shocks in times of crisis.

Evidence indicates that in high-volatility environments, such as that of financial crises, the network topology of equities markets changes, and the correlation among financial assets rises, diminishing in consequence, the effectiveness of diversification as a risk management tool. This issue is critical for strategies applied in portfolio management, where tactical and strategic asset allocation decisions are based on modeling the correlations of returns of financial assets [22, 23, 31, 32].

Nevertheless, the returns of financial assets, especially stocks, are particularly difficult to predict (see [33] for a review); however, the volatility of their returns seems to be relatively easier to forecast. The stylized fact about volatility is that it is low, but slowly decaying persistency. In this sense, it is not surprising the growing literature focused on modeling and forecasting financial volatility, due to its implications for asset pricing, portfolio management, and risk management.

One of the main problems of volatility measures is that the conditional variance is a "latent" variable. Therefore, it is not directly observable (see [34] for a discussion). There is a wide range of models to estimate this latent variable, such as autoregressive conditional heteroskedasticity (ARCH or GARCH type models) and stochastic volatility (SV) models. However, as pointed out in $[35,36]$, these models tend to fail to correctly accommodate some stylized facts regarding financial time series such as high excess kurtosis.

A novel and increasingly popular method is the quantification of realized volatility. The main advantage of this approach is that the ex-post volatility becomes necessarily observable rather than being treated as a latent variable. In this sense, it becomes straightforward to evaluate the out-ofsample forecasting accuracy of different models when predicting realized volatility, as it can be modeled directly (see $[37,38]$ for a discussion)

While our out-of-sample forecasting approach using financial networks is somewhat novel, there are recent attempts to establish relationships between local market volatilities and the international financial interlinkages. For instance, Bouri et al. [39] examine the predictive ability of commodity and significant developed stock markets forecasting the implied volatility (IV) of each one of the individuals BRICS (Brazil, Russia, India, China, and South Africa) stock markets. Using a Bayesian graphical SVAR approach (BGSVAR, see [40]), they find some evidence of Granger causality mainly from the global stock markets (and some individual markets) IV over the BRICS IV (notably, they find that the commodity market is important exclusively in South Africa. One possible explanation for this result is the strong relationship between major exporting economies and global commodity 
prices, extensively reported in [41-45]). This result is somewhat consistent with previous evidence relating to global factors and BRICS stock markets [46].

In related work, Ji et al. [47] model a dynamic network for the IV transmission among US equities, commodities, and BRICS equities. In general, they show that the integration structure of the information transmission network is somewhat unstable, with changes over time. Their results suggest that the impact of the analyzed events is heterogeneous, e.g., some events have an impact exclusively on the IV of local markets, but others impact global volatility.

In the same line, Ji et al. [48] use a directed acyclic graph to study the contemporaneous and lagged relationships between bitcoins and other financial assets as commodities, stock markets, and fixed income indices. Notably, they find little evidence of contemporaneous relations between bitcoins and other financial assets, although they find some evidence of predictability in the bear market states of bitcoins.

Respect to market volatility linkages, Aggarwal and Raja [49] study the cointegration among the stock markets of BRIC economies. Additionally, they examine the IV transmission between the Indian IV index and three international indices; in particular, they study how shocks in the IV of one market may affect other markets' volatility. Similarly, Ewing et al. [50] study the effects of the NAFTA agreement on volatility transmissions of each market. Other empirical papers exploring the linkages among financial markets include [51, 52] for NAFTA economies, [53] for linkages between the US and European markets, and [54] for Latin American markets.

Finally, Hussain Shahzad et al. [55] study spillovers in the Eurozone credit market sector. Using network theory and daily data on 14 sector-level credit default swaps (CDS) indices in the Eurozone, they identify the main sectors transmitting (and receiving) spillovers during regular periods and crisis. In particular, they find that many CDS sectors became strongly interconnected in crisis periods, and this linkage remains for some later periods, suggesting some evidence of contagion.

\section{Method}

3.1. The Minimum Spanning Tree Length (MSTL). We follow the standard procedure to obtain the return correlations and dynamic asset trees based on price market indexes $[18,56]$. The closure prices index $i$ at time date $\tau$ is $P_{i}(\tau)$. The return of index $i$ is given by $r_{i}(\tau)=\ln P_{i}(\tau)-\ln P_{i}(\tau-1)$, for a consecutive sequence of trading days. For each index $i$, daily returns are calculated within a time window of 1 month. Let be $\mathbf{r}_{i}^{t}$ the return vector of the index $i$ of the month $t$, then

$$
\rho_{i j}^{t}=\frac{\left\langle\mathbf{r}_{i}^{t} \mathbf{r}_{j}^{t}\right\rangle-\left\langle\mathbf{r}_{i}^{t}\right\rangle\left\langle\mathbf{r}_{j}^{t}\right\rangle}{\left[\left\langle\mathbf{r}_{i}^{t 2}\right\rangle-\left\langle\mathbf{r}_{i}^{t}\right\rangle^{2}\right]\left[\left\langle\mathbf{r}_{j}^{t 2}\right\rangle-\left\langle\mathbf{r}_{j}^{t}\right\rangle^{2}\right]},
$$

where $\rho_{i j}^{t}$ is the correlation coefficient between the indices $i$ and $j$ where $\langle\ldots\rangle$ indicates the average over all the trading days of the month $t$. In this way, a $N \times N$ symmetrical matrix $\mathbf{C}^{t}$ of correlations between market indexes ( $N$ is the number of financial indices) with values $-1 \leq \rho_{i j} \leq 1$.

Then, the correlations of $\mathbf{C}^{t}$ are converted to distances $d_{i j}=\left(2\left(1-\rho_{i j}\right)\right)^{(1 / 2)}$, which represents the distance between the market index $i$ and $j$. Thus, a correlation $\rho_{i j}=-1$ indicates a maximum distance of $d_{i j}=2$, whilst $\rho_{i j}=1$ indicates a minimum distance of $d_{i j}=0$.

The minimum spanning tree (hereafter, MST) is a tree structure graph that connects the $N$ indexes through $N-1$ edges avoiding loops and clicks, and where the path to connect all the nodes is minimal. The MST is constructed using the Prim algorithm [46]. In this way, MST reduces the information space of the entire network by connecting all nodes with $N(N-1) / 2$ edges, to a tree with $N-1$ edges [57].

The sum of the edges of the resulting tree $\mathbf{T}^{t}$ calculated for each month $t$ forms a time series. We define the normalized length of the MST (MSTL) as follows:

$$
L(t)=\left(\frac{1}{N-1}\right) \sum_{d_{i j}^{t} \in T^{t}} d_{i j}^{t}
$$

So, for every month, we have an MSTL. The variation in the MSTL is calculated as $\Delta L(t)=\ln L(t)-\ln L(t-1)$, which allows us to work with a stationary time series.

\subsection{The Planar Maximally Filtered Graph Length (PMFGL).} The planar maximally filtered graph (PMFG) also filters out the complete graph based on the distance matrix $\mathbf{D}^{t}$ by keeping only the main representative links by varying the genus of the graph $[58,59]$. In this case, the PMFG retains a bit more information. The MST keeps $N-1$ edges, while the PMFG keeps $3 N-6$ edges compared to the $N(N-1) / 2$ edges of the complete graph [60]. In addition, the PMFG also contains the MST.

The length of the PMFG (therefore, PMFGL) is simply defined as the sum of all distances $d_{i j}^{t}$ of the resulting distance matrix for the PMFG. Since the PMFG retains a greater number of edges (thus a greater number of correlations), it is possible that this network can better express the level of synchronization in the market. For this reason, it is included in the core models.

Since the PMFG supports cycles in the network and may include negatively correlated stocks, the PMFG length will always be greater than that of the MST. Precisely because of this feature of including more information, it is interesting to be able to compare the models that explain the risk of interconnectivity as a measure of robustness. It is worth mentioning that we do not calculate the PMFG length for regions. The PMFG includes the MST and the edges used to join the nodes in the PMFG are of minimum distance; therefore, the length of the regional PMFG is the same as the length of the MST.

3.3. Realized Variance. We measured volatility with the daily variance of stock market using the realized variance model $\left(\mathrm{RV}_{i, t}\right)[4]$ : 


$$
\mathrm{RV}_{i, t}=\sum_{j=1}^{N} r_{i, j, t}^{2} .
$$

The realized volatility is our dependent variable, where $r_{i, j, t}$ is the daily return for the day $j$ on the month $t$ for the market index $i$. We used daily data provided by Bloomberg from July 2001 to September 2019, totaling 216 months, for a total of 26 market indexes in America, Europe, Asia, and Oceania. These market indices are part of the benchmarks published by Bloomberg for each stock market at the country and region level. We included the VOX CBOE in our robustness out-of-sample test following [20] that incorporated with a control for the monthly volatility of each region and monthly variation of VIX index.

3.4. Forecasting Model and Evaluation. We used two types of forecasting models to evaluate the predictive power of the MSTL and PMFGL. First, we named "core models" at forecasting models for our in-the-sample and out-of-sample tests that include the natural logarithm variation of the MSTL (therefore, VMSTL) and include the natural logarithm variation of PMFGL (therefore, VPMFGL) (see Table 1 panels A and B). Second, for our out-of-sample tests, we named "benchmark models" at forecasting models that are inspired in a vast literature that has shown that $\mathrm{AR}(\mathrm{p})$ models are usually difficult benchmarks to beat when forecasting realized volatility $[4,8]$. In this sense, we use a heterogeneous autoregressive (HAR) model as our main benchmark (see Table 1 panel $\mathrm{C}$ ).

In the table, $\mathrm{RV}_{i, t}$ is the realized variance in the month $t$ for the market index $i, \mathrm{VMSTL}_{t-1}$ is the global minimal spin tree length in the month $t-1, \mathrm{VPMFGL}_{t-1}$ is the global planar maximally filtered graph in the month $t-1, \mathrm{RV}_{i, t-1}$, $\mathrm{RV}_{i, t-2}$, and $\mathrm{RV}_{i, t-3}$ are the first, second, and third lags of the realized volatility, respectively, for the market index $i$, and $e_{i}$ is the disturbance error.

Our main goal in this paper is to test the existence of Granger causality from the structure of the network to the realized volatility. For this, we focus on testing the following null hypothesis $H_{0}: \beta_{i}=0$; this means that we are comparing our core models to benchmark models (see Table 1). Our null hypothesis both in sample and out of sample posits that the VMSTL $\mathrm{VM}_{t-1}$ and VPMFGL $\mathrm{V}_{t-1}$ have no role in predicting the market index realized volatility. We test these hypotheses both in sample and out of sample focusing on one-step-ahead forecasts only, leaving the analysis of multistep-ahead forecasts as an extension for future research.

In-sample evaluations are carried out using the $t$-statistic associated with the coefficient of the minimal spin tree length. For covariance stationary processes, the central limit theorem requires a proper estimation of the long-run variance; in this sense, we use HAC standard errors as suggested in $[61,62]$ (Newey and West [61] propose a Barlett kernel to ensure a positive definite variance matrix. Additionally, Newey and West [62] propose an automatic lag selection method for the covariance matrix estimation). In-sample estimates, however, are usually criticized because they are relatively different from a real-time forecasting exercise and also because there are prone to data mining-induced overfitting. To mitigate these shortcomings, we also consider out-of-sample analysis.

For out-of-sample evaluations, as we are working in an environment with nested models, we use the ENCNEW test proposed in [63] (other tests for nested models such as [64-66] were also considered with similar messages, and they are available upon request). Again, for out-of-sample analysis, we are considering the null hypothesis $H_{0}: \beta_{i}=0$. In the context of linear models estimated by OLS, Clark and McCracken [63] derive the correct asymptotic distribution of this test. While the distribution is not standard, critical values for one-step-ahead forecasts are available in their paper. Under general conditions, the asymptotic distribution of the ENCNEW test is a functional of Brownian motions depending on the number of excess parameters of the nesting model, which is 1 in our specifications, and on the parameter $\pi$ defined as the limit of the ratio $P / R$, where $P$ is the number of one-step-ahead forecasts and $R$ is the size of the first expanding window used in the out-of-sample analysis $(\pi$ is defined as the limit $P / R$ when $P, R \longrightarrow \infty$. Clark and McCracken [63] show that the asymptotic distribution of the ENCNEW depends, among other parameters, on $\pi$. In this sense, $\pi=0.4$ can be interpreted as the estimation window being approximate twice the prediction window's length). The asymptotic distribution of the test varies also with the scheme used to update the estimates of the parameters: either rolling, recursive, or fixed. Additionally, we emphasize that this test is one-sided, and in other words, rejection of the null occurs only when the statistic is greater than a critical value located at the right tail of the distribution (see $[67,68]$ for wonderful reviews and further details about the implementation of out-of-sample tests of predictive ability in nested model environments).

For in-sample analysis, we estimate our models with all the available observations. For the case of out-of-sample analysis, we split the sample in three different ways, as suggested in [69]. For each splitting, we consider two windows: an initial estimation window of size $R$ and an evaluation window of size $P$ such that $T=P+R$, where $T$ is the total number of observations. We split the samples in three different ways. First, we use the first half for the initial estimations and the second half to make our predictions. Second, we use approximately the first third of observations for initial estimations and two thirds for evaluation. Third, we use approximately $70 \%$ of initial observations for estimation and $30 \%$ for evaluation. Finally, we update our parameters using recursive windows, although results with rolling windows are very similar. We only report the results for the third division $(\pi=0.4)$ for saving space; however, the message of predictability is very similar in all divisions and they are available upon request.

3.5. The Data. We used daily data provided by Bloomberg from July 2001 to September 2019, totaling 223 months, for a total of 26 market indexes in North America, Latin America, Europe, Asia, and Oceania (see Table 2 for details). These indices belong to regional stock indices published by 
TABLE 1: The main econometric models for both the core and benchmark models.

Panel A: in-the-sample core models

(1) $\mathrm{RV}_{i, t}=c+\beta_{i} * \mathrm{VPMFGL}_{t-1}+\gamma_{i, 1} * \mathrm{RV}_{i, t-1}+\gamma_{i, 2} * \mathrm{RV}_{i, t-2}+\gamma_{i, 3} * \mathrm{RV}_{i, t-3}+e_{i}$

(2) $\mathrm{RV}_{i, t}=c+\beta_{i} * \mathrm{VMSTL}_{t-1}+\gamma_{i, 1} * \mathrm{RV}_{i, t-1}+\gamma_{i, 2} * \mathrm{RV}_{i, t-2}+\gamma_{i, 3} * \mathrm{RV}_{i, t-3}+e_{i}$

Panel B: out-of-sample core models

(3) $\mathrm{RV}_{i, t}=c+\beta_{i} * \mathrm{VPMFGL}_{t-1}+\gamma_{i, 1} * \mathrm{RV}_{i, t-1}+\gamma_{i, 2} * \mathrm{RV}_{i, t-2}+\gamma_{i, 3} * \mathrm{RV}_{i, t-3}+\gamma_{i, 4} * \mathrm{RV}_{i, t-4}+\gamma_{i, 5} * \mathrm{RV}_{i, t-5}+\gamma_{i, 6} * \mathrm{RV}_{i, t-6}+e_{i}$

(4) $\mathrm{RV}_{i, t}=c+\beta_{i} * \mathrm{VMSTL}_{t-1}+\gamma_{i, 1} * \mathrm{RV}_{i, t-1}+\gamma_{i, 2} * \mathrm{RV}_{i, t-2}+\gamma_{i, 3} * \mathrm{RV}_{i, t-3}+\gamma_{i, 4} * \mathrm{RV}_{i, t-4}+\gamma_{i, 5} * \mathrm{RV}_{i, t-5}+\gamma_{i, 6} * \mathrm{RV}_{i, t-6}+e_{i}$

(5) $\mathrm{RV}_{i, t}=c+\beta_{i} * \mathrm{VPMFGL}_{t-1}+\gamma_{i, 1} * \mathrm{RV}_{i, t-1}+\gamma_{i, 2} * \mathrm{RV}_{i, t-2}+\gamma_{i, 3} * \mathrm{RV}_{i, t-3}+e_{i}$

(6) $\mathrm{RV}_{i, t}=c+\beta_{i} * \mathrm{VMSTL}_{t-1}+\gamma_{i, 1} * \mathrm{RV}_{i, t-1}+\gamma_{i, 2} * \mathrm{RV}_{i, t-2}+\gamma_{i, 3} * \mathrm{RV}_{i, t-3}+e_{i}$

(7) $\mathrm{RV}_{i, t}=c+\beta_{i} * \mathrm{VPMFGL}_{t-1}+\gamma_{i, 1} * \mathrm{RV}_{i, t-1}+\gamma_{i, 2} * \mathrm{RV}_{i, t-2}+\gamma_{i, 3} * \mathrm{RV}_{i, t-3}+\delta_{i} * \mathrm{VVIX}_{t-1}+e_{i}$

(8) $\mathrm{RV}_{i, t}=c+\beta_{i} * \mathrm{VMSTL}_{t-1}+\gamma_{i, 1} * \mathrm{RV}_{i, t-1}+\gamma_{i, 2} * \mathrm{RV}_{i, t-2}+\gamma_{i, 3} * \mathrm{RV}_{i, t-3}+\delta_{i} * \mathrm{VVIX}_{t-1}+e_{i}$

Panel C: out-of-sample benchmark models

(9) $\mathrm{RV}_{i, t}=c+\gamma_{i, 1} * \mathrm{RV}_{i, t-1}+\gamma_{i, 2} * \mathrm{RV}_{i, t-2}+\gamma_{i, 3} * \mathrm{RV}_{i, t-3}+\gamma_{i, 4} * \mathrm{RV}_{i, t-4}+\gamma_{i, 5} * \mathrm{RV}_{i, t-5}+\gamma_{i, 6} * \mathrm{RV}_{i, t-6}+e_{i}$

(10) $\mathrm{RV}_{i, t}=c+\gamma_{i, 1} * \mathrm{RV}_{i, t-1}+\gamma_{i, 2} * \mathrm{RV}_{i, t-2}+\gamma_{i, 3} * \mathrm{RV}_{i, t-3}+e_{i}$

(11) $\mathrm{RV}_{i, t}=c+\gamma_{i, 1} * \mathrm{RV}_{i, t-1}+\gamma_{i, 2} * \mathrm{RV}_{i, t-2}+\gamma_{i, 3} * \mathrm{RV}_{i, t-3}+\delta_{i} * \mathrm{VVIX}_{t-1}+e_{i}$

Source: authors' elaboration.

TABLE 2: Stock country indices by region.

\begin{tabular}{lr}
\hline Region & Indices \\
\hline $\begin{array}{l}\text { North } \\
\text { America }\end{array}$ & S\&P500 and NASDAQ from USA and TSX from Canada \\
Latin America & IPC from Mexico, IBOVESPA from Brazil, IPSA from Chile, MERVAL from Argentina, and IGBVL from Peru \\
Europe & FTSE from UK, CAC from France, DAX from Germany, IBEX from Spain, MIB from Italy, AEX from Holland, OMX from \\
Sweden, RTS from Russia, and SMI from Swiss & Swed \\
Asia & NIKKEI from Japan, HANG-SENG from Hong Kong, KOSPI from Korea, TSE from Taiwan, JSE from Indonesia, KLCI \\
Oceania & from Malaysia, and ST from Singapore \\
\hline
\end{tabular}

Source: authors' elaboration.

Bloomberg for each stock market at the country and region level. As mentioned above, we included the CBOE VIX index in our robustness section as part of an out-of-sample test following Lavin et al. [20], who incorporated the monthly variation of the VIX index as a control for the monthly volatility of each regional market.

\section{Empirical Results}

In this section, we first report the estimation results of 26 market indices of our core models (Table 1 panel A) using in-sample data. Secondly, we evaluate the forecasting performance with our benchmark models (Table 1 panel B). Finally, we check the robustness of models adding in the out-of-sample test a lag of variation of VIX (VVIX). We calculate the ENCNEW out-of-sample test of Clark and McCracken [63].

Figure 1 shows a representation of the financial indices' MSTs in three different periods: before, during, and after the financial crisis of 2008. The proximity of the assets about their origin geographical location remains an unalterable property through time. This phenomenon produces clusters based on geographical location. For example, Asian market indices tend to cluster together. The same is true of European indices. Only the two Oceania indices (Australia and New Zealand) appear to be accommodating regardless of geographic location. However, the lengths of the MSTs are different. The MST length was 10.97 in precrisis, and it decreased to 10.23 in crisis and then increased again to 11.68 in the postcrisis period. In times of financial crisis, markets synchronize, increasing the intercorrelations between financial assets. Consequently, the distances represented on each edge of the network shorten [18].

4.1. In-Sample Analysis. Tables 3-5 report estimates of core models in Table 1 panel A. In all Tables 3-5, we consider monthly frequencies and use HAC standard errors according to $[61,62]$. Generally speaking, the VMSTL coefficients are more significant than the PMFG coefficients. This evidence shows that the MSTL is a more efficient measure because the additional information included in the PMFGL does not represent a higher statistical significance. When comparing the significance of the core models (1) and (2)fd 2 presented in Table 1 panel A, we observed that the MSTL has greater predictive power, presenting statistical significance in 11 markets out of 26 in total to the PMFG that shows statistical significance in 7 markets out of 26 in total, consistent with the idea that the MSTL is more efficient by not considering correlations of lesser magnitude.

However, the predictive power of VMSTL varies by geographic area. In specific, the VMSTL coefficient is significant at least at a $10 \%$ level in most European and Asian stock markets (see Tables 4 and 5), but we did not find significance in the VMSTL coefficient in the models that represent the American equity markets (see Table 3 ). These results imply the existence of causality to the Granger between the dynamics of the network of correlations formed 


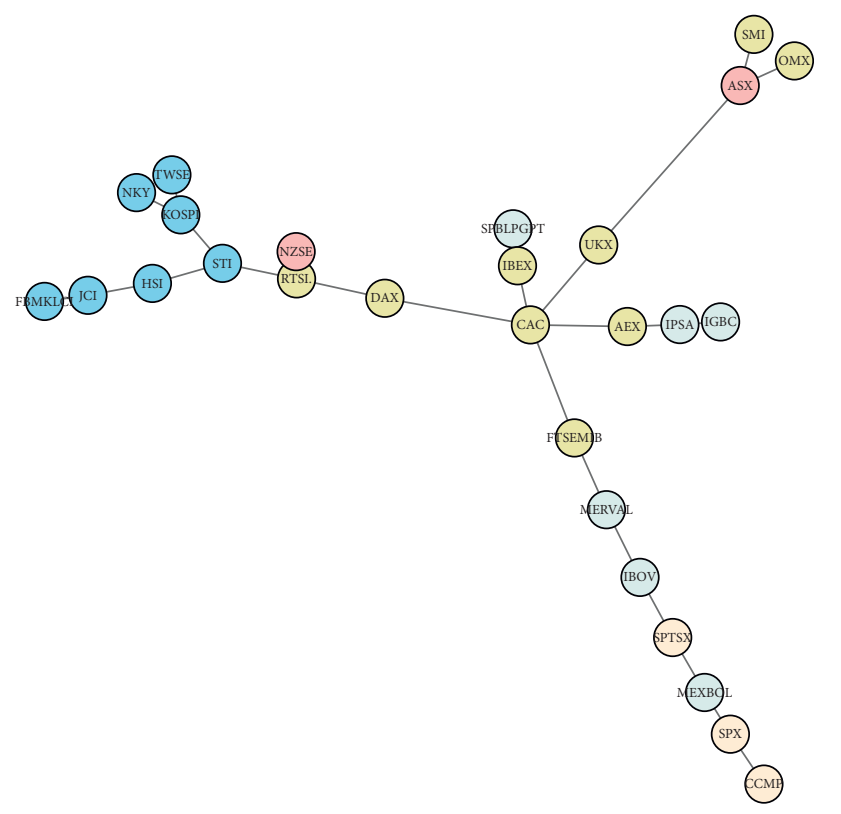

(a)

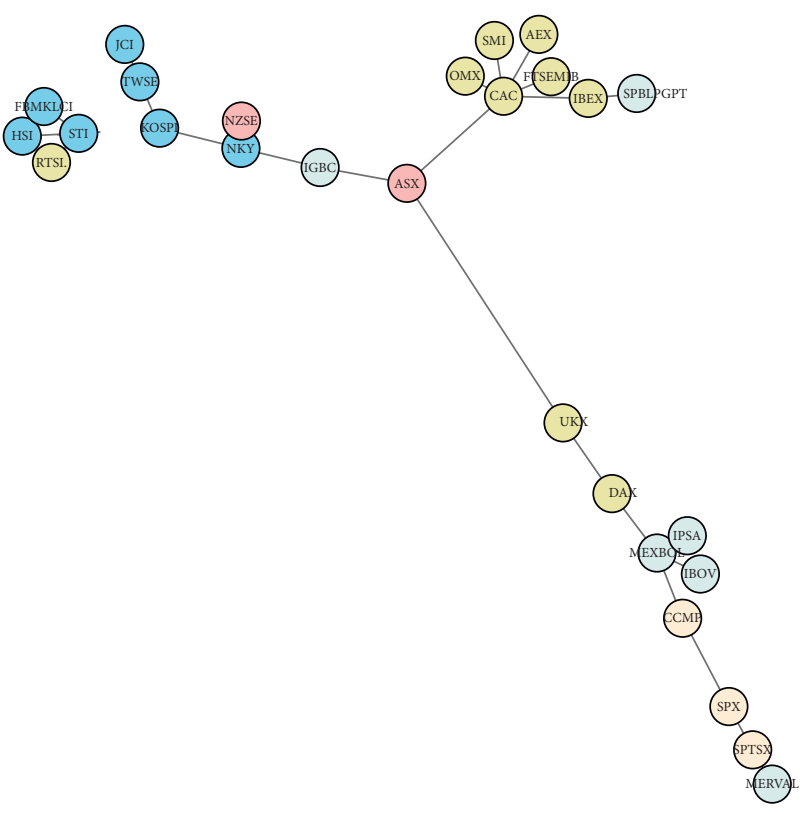

(b)

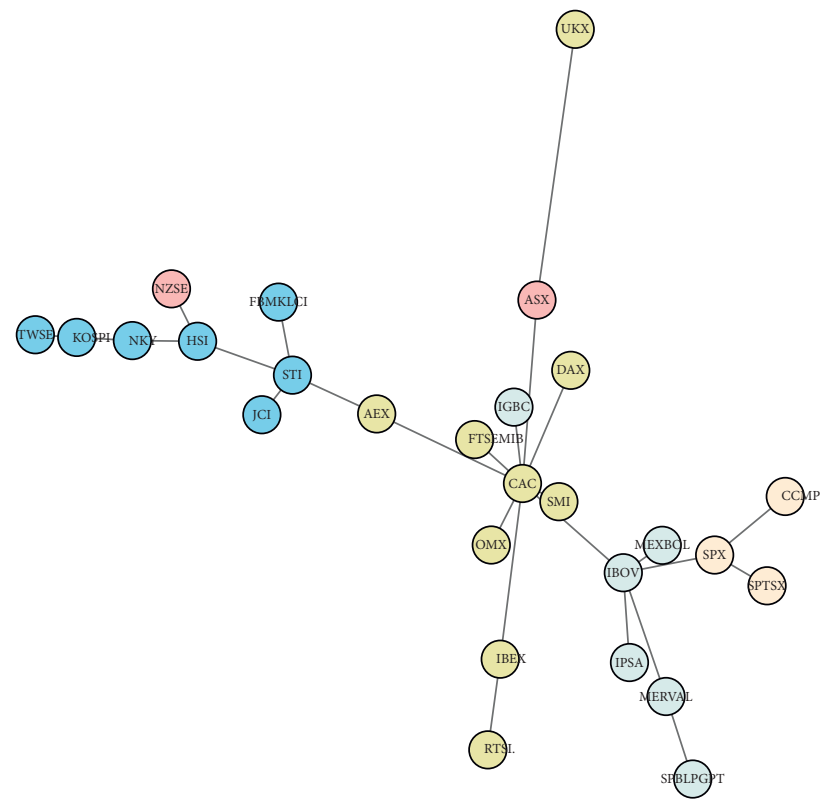

(c)

Figure 1: The resulting MSTs for three different periods. (a) Precrisis (January, February, and March 2008), (b) crisis (September, October, and November 2008), and (c) postcrisis (March, April, and May 2009). The color of the vertex represents different regions: blue: Asia, light blue: Latin America, light yellow: Europe, light Salmon: North America, and pink: Oceania.

between global stock markets and the volatility of the European and Asian stock markets.

Regarding Europe, Table 4 shows that the volatility of the stock markets in France $(\beta=-0.672, p=0.015)$, Spain $(\beta=-0.700, p=0.0 .044)$, Italy $(\beta=-0.838, p=0.0124)$, and Sweden $(\beta=-0.822, p=0.0214)$ has the highest relationship with the global network of correlations lagging in one month. Regarding Asia, Table 5 indicates that the volatility realized in the markets of Taiwan $(\beta=-1.083, p=0.001)$, Korea $(\beta=-0.829, p=0.0188)$, and Hong Kong $(\beta=-0.672$, $p=0.0476)$ shows the relationship with the global network of correlations lagging in a month of greater magnitude of statistical significance. Regarding Oceania, Table 5 shows that only the volatility of the New Zealand stock market $(\beta=-0.623, p=0.0474)$ shows statistical significance with the global network of correlations with one month of lag.

Several additional features are worth noticing from our in-sample core models. First, the term of the constant and the coefficient of the first volatility lag are statistically significant in all markets (see Tables 3-5), consistent with the strong autocorrelation of the volatility of the equity indices. Second, the coefficient of the three lags is positive in all 


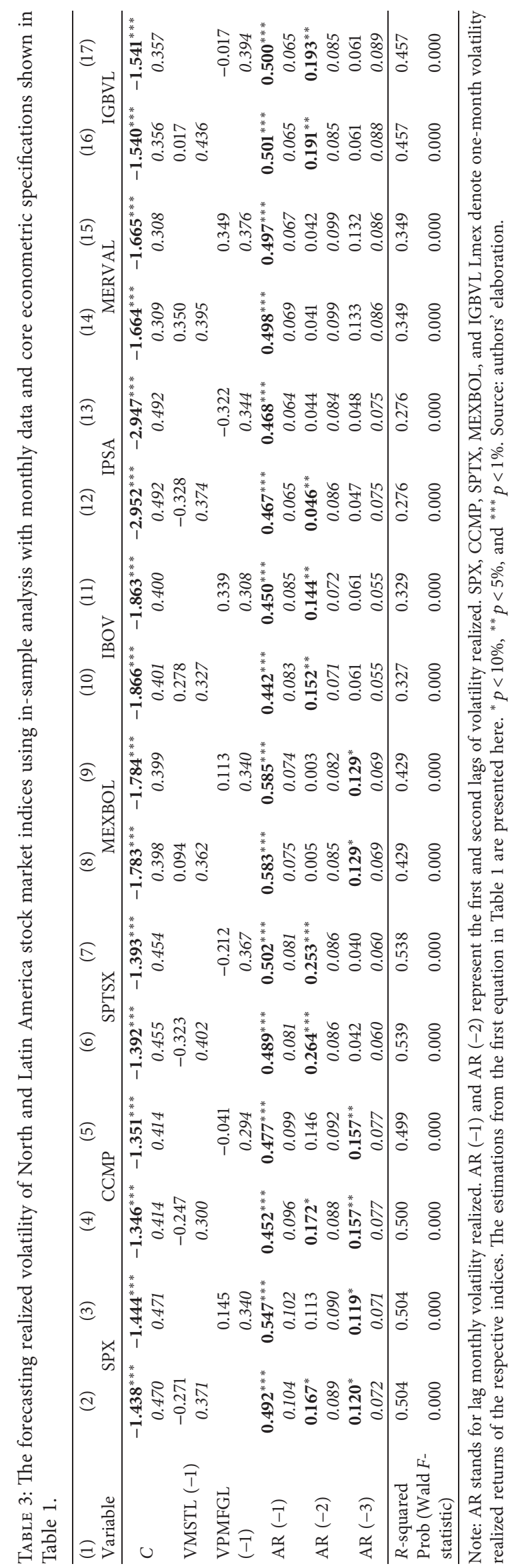




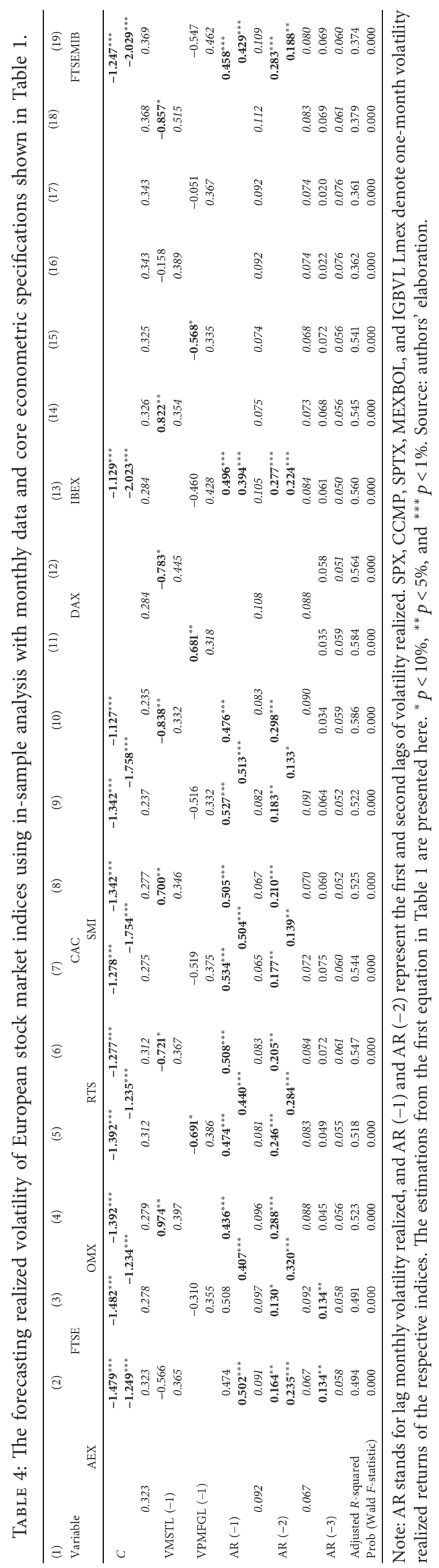




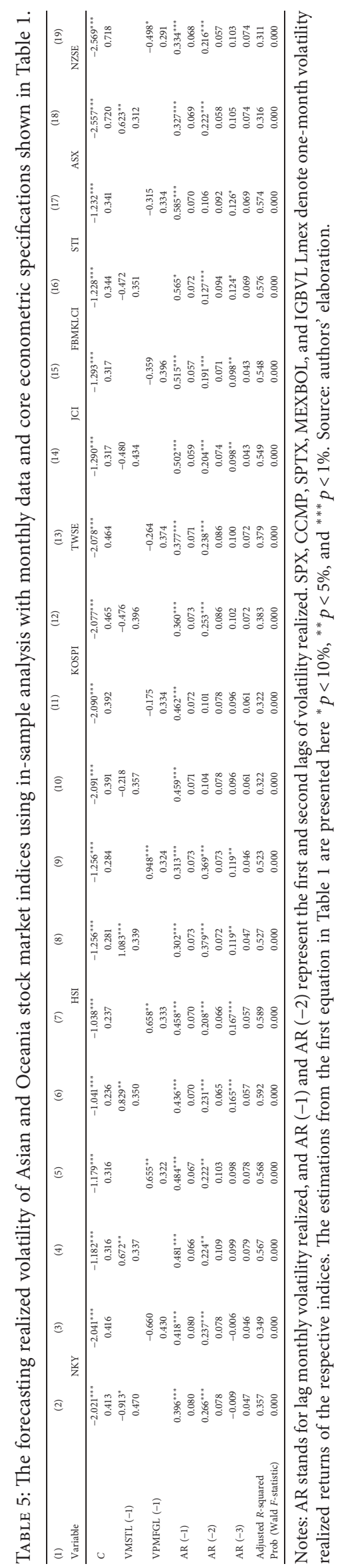




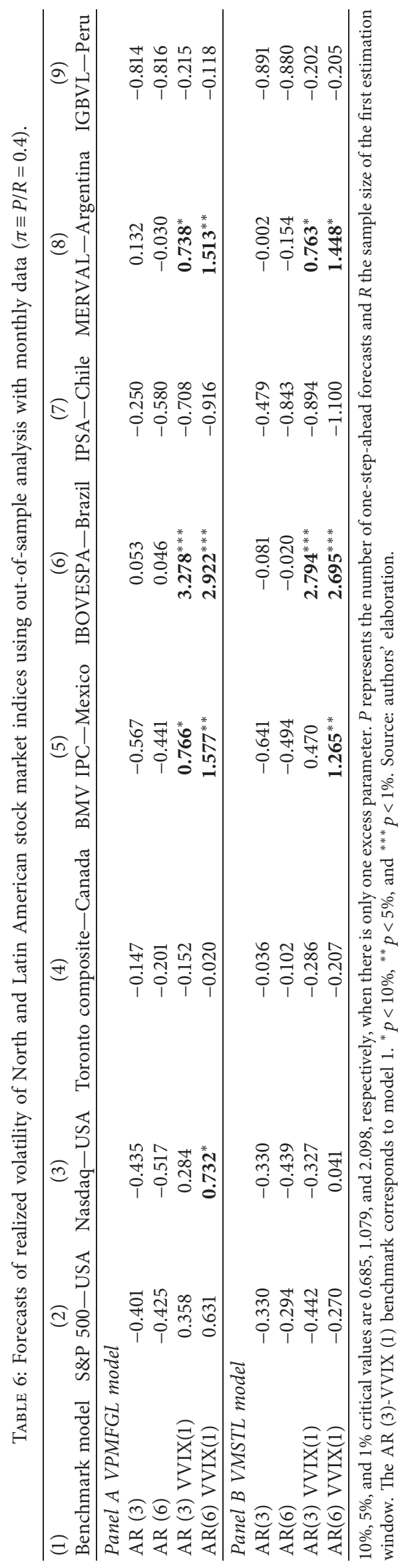




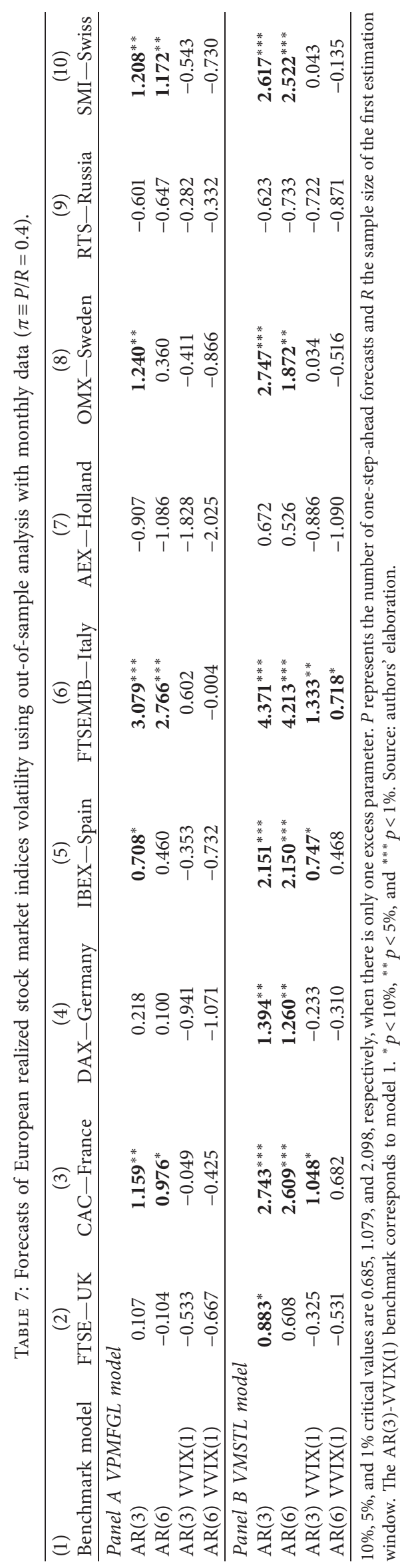




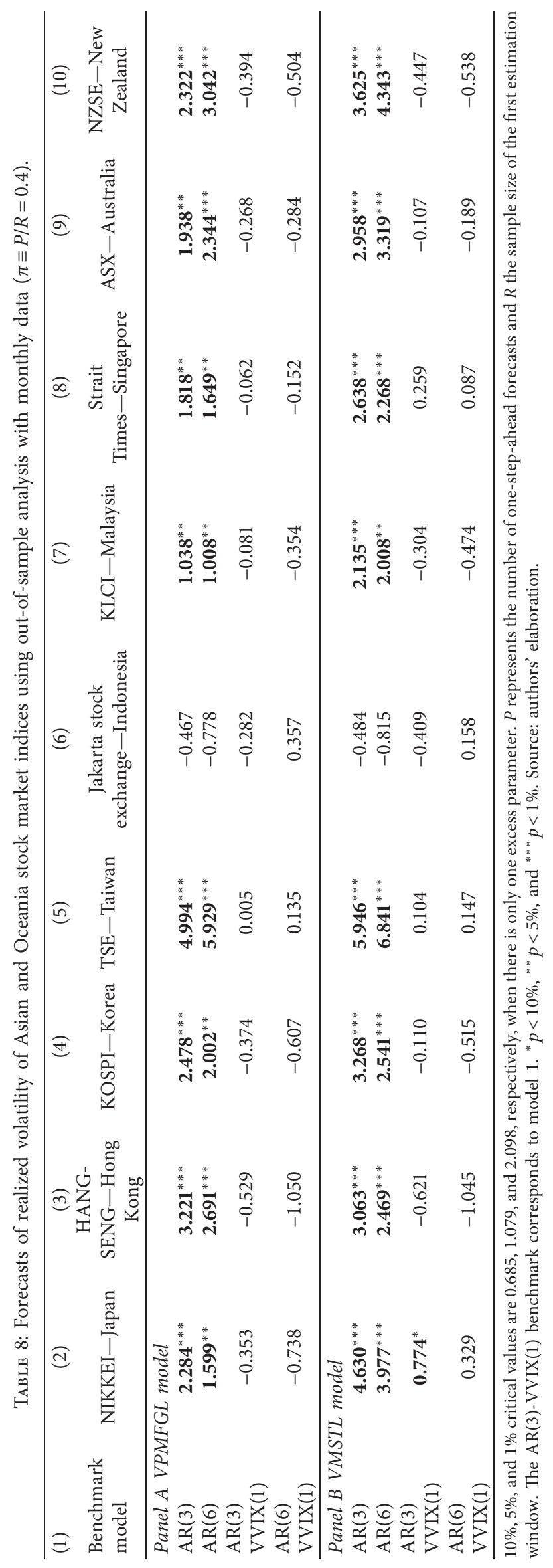


markets, consistent with the persistence of financial markets: an increase in volatility is an indicator of an increase in volatility in the following period. This relationship is consistent with the first lag in all markets except the UK, but statistical significance decreases for the second and third lags. Finally, the adjusted determination coefficients are between $27.6 \%$ and $59.2 \%$, the highest being the KOSPI in Korea and the lowest the SPIPSA in Chile.

4.2. Out-of-Sample. Tables 6-8 show the ENCNEW test results [63] in out-of-sample exercise for the Americas, Europe, and Asia-Oceanic. These tables focus on the core models described in Table 1 panel $\mathrm{B}$, and the results correspond to the statistical difference between the core models presented in Table 1 panel B (with VMSTL and VPMFGL) versus the benchmark models presented in Table 1 panel $\mathrm{C}$ when the number of observations to make the forecast is $40 \%$ of the total sample $(P / R=0.4)$.

In Tables 6-8, we use the following notation to describe specifications in Table 1: AR (3) and AR (6) denote an autoregressive process of orders 3 and 6, respectively, for the one-period return in realized volatility stock indices. VVIX (1) denotes a lag effect of the monthly variation of the VIX, in order to add a robustness test to the results. Additionally, we separate Tables 6-8 into two panels: panel A in Tables 6-8 reports the comparison of the core model (Table 1 panel B) that includes the lagged variation of the PMFGL in one month with our benchmark models (Table 1 panel C); panel $\mathrm{B}$ in Tables 6-8 reports the comparison of the core model (Table 1 panel $\mathrm{B}$ ) that includes the lagged variation in a period of the MSTL with the benchmark models (Table 1 panel C)

In the case of America (Table 6), only the markets of Mexico, Brazil, and Argentina reject the null hypothesis for the VVIX benchmark models. In Brazil's case, the probability of rejecting the null hypothesis is greater than $1 \%$, which means that the forecast model incorporates the lag of the variation of the MSTL, and the lag of the variation of the PMFGL is statistically better than the VVIX benchmarks models. This result is repeated for Mexico and Argentina, although with a significance level that fluctuates between 5\% and $10 \%$.

Regarding Europe, the core models (incorporating the one-month lag variation of the MSTL and PMFGL) obtain better results compared to all the benchmark models. In general terms, we observe that the MSTL has greater predictive power than the PMFGL, which turns out to be significant in 7 out of 9 European markets considered, while the model that considers the PMFGL only turns out to be significant in 5 out of 9 markets. Panel B of Table 7 shows the results of the model comparison that includes the lagged variation of the MSTL.

We present similar results in Asia and Oceania. Table 8 shows that $8 / 9$ market indices reject the null hypothesis, all over the $5 \%$ levels, indicating that both the variation of the PMFGL lagging in one period and the variation of the MSTL lagging in a period add useful information for improving the forecast of benchmark models. However, when comparing with a benchmark model that incorporates the VVIX lagging in a period, the significance of the proposed models disappears, indicating a strong influence of the VIX on the volatility of the Asian and Oceania economies.

\section{Conclusions}

The interconnectedness among financial institutions plays a crucial role in terms of the systemic risk of financial markets. This issue is especially critical during financial turmoil when uncertainty and volatility spur. The recent financial network literature has been studying this phenomenon, focusing mainly on situations where a shock over a single or a group of critical nodes could destabilize the entire system [70]. Independently of the nature of the shocks, its consequences are clear in terms of volatility on financial markets, especially on stock markets and their time-varying dependence structure [71].

In this research, we explore a different view. As the economic and financial literature states, uncertainty provokes a negative impact on economic growth, expectations, investment, consumption, and future returns on stock markets. For this reason, we analyze network theory's application as a tool to forecast the volatility of stock markets. In this sense, improving the actual forecasting models in terms of performance and accuracy will benefit practitioners, regulators, and academics in their tasks.

We contribute to the extant literature in two ways. On the first hand, we help to improve the actual forecasting models in terms of performance and accuracy, which will benefit practitioners, regulators, and academics in the task of financial market regulation, crisis monitoring, portfolio management, risk strategies, and asset pricing, among other roles. On the other hand, we establish a bridge between two important fields: financial network and forecasting literature. This paper could generate more significant developments in both areas, enhancing the quality of empirical analysis of financial markets and gaining a broad view of complex systems.

Our results evidence that it is possible to improve actual forecasting volatility models through network metrics for many stock indices. Using assets' correlation networks, we show that the forecasting accuracy and performance of realized volatility standard models enhance, even including volatility gauges such as CBOE VIX index as a control variable. This outcome is a novel contribution from the financial networks' literature to the economic and financial areas to develop future contributions and connections between these two relevant fields.

These results have two implications. From a practitioner's point of view, it is possible to use these network measures to assess current and future diversification possibilities and forecast the portfolios' expected volatilities, improving consequently, delegated investment services. Secondly, these results are useful for regulatory agents, who monitor the state of the financial system, its stability, and its systemic risk. 
Our research contributes to enhancing the forecasting capabilities of volatility in financial markets based on market data. From a policy point of view, it is highly desirable that Central Banks, Stocks Exchanges, and the investment community, in general, advance in the task of improving the estimations of volatility. For instance, whether an independent institution monitors and publishes future volatility prospects, the evolution of asset synchronization, and asset diversification capabilities will help agents better assess risk and return relationships in the financial markets, with its consequently positive impact on the financial efficiency. In this sense, this work would help policymakers improve financial stability frameworks and design models that consider financial markets a complex system, i.e., their underlying structure, their correlations, and their interactions.

We built the edges of the complete network based on Pearson correlations between returns of different stock indices. These correlations measure the linear association between them, leaving aside a more complex nonlinear component that determines the covariation between different elements of this system. A possible extension of this work is to capture these nonlinearities through more sophisticated methods inferring the network's structural connectivity [72].

Likewise, the presence of high-frequency cross-correlations between synchronous time evolution of stock markets is known. It is also known that when considering higher frequencies (not only across one day-trading), there are changes in the stocks' hierarchical structures [25]. This idea makes us think that the realized volatility can be expressed differently according to the frequency used in the analysis, and consequently, it would be necessary to study the behavior of our volatility models proposed in these scenarios.

\section{Data Availability}

The data used to support the findings of this study are available from the corresponding author upon request.

\section{Conflicts of Interest}

The authors declare that they have no conflicts of interest regarding the publication of this paper.

\section{Acknowledgments}

The authors would like to thank Universidad Adolfo Ibáñez under grant Internal Funds for Research 1154-2020 (Business School-Jaime F. Lavin) for supporting this research.

\section{References}

[1] N. Antonakakis, I. Chatziantoniou, and G. Filis, "Dynamic comovements of stock market returns, implied volatility and policy uncertainty," Economics Letters, vol. 120, no. 1, pp. 87-92, 2013.

[2] G. M. Gallo and E. Otranto, "Volatility transmission in financial markets: a new approach," 2005, http://www.ds.unifi. it/ricerca/pubblicazioni/working_papers/2005/wp2005_10. pdf.

[3] K. Yang, L. Chen, and F. Tian, "Realized volatility forecast of stock index under structural breaks," Journal of Forecasting, vol. 34 , no. 1, pp. 57-82, 2015.

[4] H. Wang, "VIX and volatility forecasting: a new insight," Physica A: Statistical Mechanics and Its Applications, vol. 533, Article ID 121951, 2019.

[5] T. G. Andersen, T. Bollerslev, and N. Meddahi, "Correcting the errors: volatility forecast evaluation using high-frequency data and realized volatilities," Econometrica, vol. 73, no. 1, pp. 279-296, 2005.

[6] T. Bollerslev, R. F. Engle, and D. B. Nelson, "Chapter 49 arch models," Handbook of Econometrics, vol. 4, pp. 2959-3038, 1994.

[7] T. G. Andersen, T. Bollerslev, F. X. Diebold, and P. Labys, "Modeling and forecasting realized volatility," Econometrica, vol. 71, no. 2, pp. 579-625, 2003.

[8] F. Corsi, "A simple approximate long-memory model of realized volatility," Journal of Financial Econometrics, vol. 7, no. 2, pp. 174-196, 2009.

[9] T. Bury, "A statistical physics perspective on criticality in financial markets," Journal of Statistical Mechanics: Theory and Experiment, vol. 11, p. 11004, 2013.

[10] N. Kiyotaki and J. Moore, "Credit cycles," Journal of Political Economy, vol. 105, no. 2, pp. 211-248, 1997.

[11] F. Allen and D. Gale, "Financial markets, intermediaries, and intertemporal smoothing," Journal of Political Economy, vol. 105, no. 3, pp. 523-546, 1997.

[12] R. Albert, H. Jeong, and A.-L. Barabasi, "Erratum: correction: error and attack tolerance of complex networks," Nature, vol. 409, no. 6819, p. 542, 2001.

[13] B. Porterie, K. Ahmed, J.-P. Clerc, N. Zekri, and L. Zekr, "Universal scaling of forest fire propagation," 2008, https:// arxiv.org/abs/0805.3365.

[14] A. Haldane, "Rethinking the financial network," 2009.

[15] A. G. Haldane and V. Madouros., The Dog and the Frisbee BIS Central Bankers' Speech at Federal Reserve Bank of Kansas City's 36th Economic Policy Symp, The Changing Policy Landscape, Jackson Hole, WY, USA, 2012.

[16] R. N. Mantegna, "Hierarchical structure in financial markets," The European Physical Journal B, vol. 11, no. 1, pp. 193-197, 1999.

[17] J. Eberhard, J. F. Lavin, and A. Montecinos-Pearce, "A network-based dynamic analysis in an equity stock market," Complexity, vol. 2017, Article ID 3979836, 16 pages, 2017.

[18] J. P. Onnela, A. Chakraborti, K. Kaski, J. Kertesz, and A. Kanto, "Dynamics of market correlations: taxonomy and portfolio analysis," Physical Review E, vol. 68, no. 5, Article ID 056110, 2003.

[19] R. N. Mantegna and H. Eugene Stanley, Introduction to Econophysics: Correlations and Complexity in Finance, Cambridge University Press, Cambridge, UK, 1999.

[20] J. F. Lavin, M. A. Valle, and N. S. Magner, "Modeling overlapped mutual funds' portfolios: a bipartite network approach," Complexity, vol. 2019, Article ID 1565698, 20 pages, 2019.

[21] H.-L. Gao and D.-C. Mei, "The correlation structure in the international stock markets during global financial crisis," Statistical Mechanics and Its Applications, vol. 534, Article ID 122056, 2019.

[22] T. Výrost, Š. Lyócsa, and E. Baumöhl, "Network-based asset allocation strategies," The North American Journal of Economics and Finance, vol. 47, pp. 516-536, 2019. 
[23] W. Li, U. Hommel, and S. Paterlini, "Network topology and systemic risk: evidence from the Euro Stoxx market," Finance Research Letters, vol. 27, pp. 105-112, 2018.

[24] R. Coelho, S. Hutzler, P. Repetowicz, and P. Richmond, "Sector analysis for a FTSE portfolio of stocks," Physica A: Statistical Mechanics and Its Applications, vol. 373, pp. 615626, 2007.

[25] G. Bonanno, F. Lillo, and R. N. Mantegna, "High-frequency cross-correlation in a set of stocks," Quantitative Finance, vol. 1, no. 1, pp. 96-104, 2001.

[26] D. Acemoglu, A. Ozdaglar, and A. Tahbaz-Salehi, "Systemic risk and stability in financial networks," American Economic Review, vol. 105, no. 2, pp. 564-608, 2015.

[27] S. J. Koopman, B. Jungbacker, and E. Hol, "Forecasting daily variability of the S\&P 100 stock index using historical, realised and implied volatility measurements," Journal of Empirical Finance, vol. 12, no. 3, pp. 445-475, 2005.

[28] A. Clements and Y. Liao, "Forecasting the variance of stock index returns using jumps and cojumps," International Journal of Forecasting, vol. 33, no. 3, pp. 729-742, 2017.

[29] F. Ferriani and P. Zoi, "The dynamics of price jumps in the stock market: an empirical study on Europe and U.S," The European Journal of Finance, vol. 26, pp. 1-25, 2020.

[30] S. Wälti, "Stock market synchronization and monetary integration," Journal of International Money and Finance, vol. 30, no. 1, pp. 96-110, 2011.

[31] J. Cai, F. Eidam, A. Saunders, and S. Steffen, "Syndication, interconnectedness, and systemic risk," Journal of Financial Stability, vol. 34, pp. 105-120, 2018.

[32] G. Peralta and A. Zareei, "A network approach to portfolio selection," Journal of Empirical Finance, vol. 38, pp. 157-180, 2016.

[33] D. Rapach and G. Zhou, "Forecasting stock returns," Handbook of Economic Forecasting, vol. 2, pp. 328-383, Elsevier, Amsterdam, Netherlands, 2013.

[34] M. McAleer and M. C. Medeiros, "Realized volatility: a review," Econometric Reviews, vol. 27, no. 1-3, pp. 10-45, 2008.

[35] T. Bollerslev, "A conditionally heteroskedastic time series model for speculative prices and rates of return," The Review of Economics and Statistics, vol. 69, no. 3, p. 542, 1987.

[36] H. Malmsten and T. Teräsvirta, "Stylized facts of financial time series and three popular models of volatility," SSE/EFI Working Paper Series in Economics and Finance, vol. 563, pp. 1-44, Stockholm School of Economics, Stockholm, Sweden, 2004.

[37] F. M. Bandi and J. R. Russell., "Separating microstructure noise from volatility," Journal of Financial Economics, vol. 79, no. 3, pp. 655-692, 2006.

[38] E. Maasoumi and M. McAleer, "Realized volatility and long memory: an overview," Econometric Reviews, vol. 27, no. 1-3, pp. 1-9, 2008.

[39] E. Bouri, R. Gupta, S. Hosseini, and C. K. M. Lau, "Does global fear predict fear in BRICS stock markets? Evidence from a Bayesian graphical structural VAR model," Emerging Markets Review, vol. 34, pp. 124-142, 2018.

[40] D. F. Ahelegbey, M. Billio, and R. Casarin, "Bayesian graphical models for structural vector autoregressive processes," Journal of Applied Econometrics, vol. 31, no. 2, pp. 357-386, 2016.

[41] Y.-C. Chen, K. Rogoff, and B. Rossi, "Predicting agri-commodity prices: an asset pricing approach," SSRN Electronic Journal, Article ID 1616853, 2010.

[42] B. Rossi, "The changing relationship between commodity prices and equity prices in commodity exporting countries," IMF Economic Review, vol. 60, no. 4, pp. 533-569, 2012.
[43] P. Pincheira and N. Hardy, "Forecasting base metal prices with the Chilean exchange rate," Resources Policy, vol. 62, pp. 256-281, 2019.

[44] P. M. Pincheira and N. Hardy, "Forecasting aluminum prices with commodity currencies," SSRN Electronic Journal, Article ID 3511564, 2019.

[45] P. M. Pincheira and N. Hardy, "The predictive relationship between exchange rate expectations and base metal prices," SSRN Electronic Journal, Article ID 3263709, 2018.

[46] W. Mensi, S. Hammoudeh, J. C. Reboredo, and D. K. Nguyen, "Do global factors impact BRICS stock markets? A quantile regression approach," Emerging Markets Review, vol. 19, pp. 1-17, 2014.

[47] Q. Ji, E. Bouri, and D. Roubaud, "Dynamic network of implied volatility transmission among US equities, strategic commodities, and BRICS equities," International Review of Financial Analysis, vol. 57, pp. 1-12, 2018.

[48] Q. Ji, E. Bouri, R. Gupta, and D. Roubaud, "Network causality structures among Bitcoin and other financial assets: a directed acyclic graph approach," The Quarterly Review of Economics and Finance, vol. 70, pp. 203-213, 2018.

[49] S. Aggarwal and A. Raja, "Stock market interlinkages among the BRIC economies," International Journal of Ethics and Systems, vol. 35, no. 1, pp. 59-74, 2019.

[50] T. Ewing, J. E. Payne, and B. Clifford Sowell, “Transmission of conditional stock return volatility across North American markets: evidence from pre-and post-NAFTA," The International Trade Journal, vol. 15, no. 4, pp. 409-427, 2001.

[51] R. Aggarwal and N. A. Kyaw, "Equity market integration in the NAFTA region: evidence from unit root and cointegration tests," International Review of Financial Analysis, vol. 14, no. 4, pp. 393-406, 2005.

[52] B. T. Ewing, J. E. Payne, and C. Sowell, "NAFTA and North American stock market linkages: an empirical note," The North American Journal of Economics and Finance, vol. 10, no. 2, pp. 443-451, 1999.

[53] A. Kanas, "Linkages between the US and European equity markets: further evidence from cointegration tests," Applied Financial Economics, vol. 8, no. 6, pp. 607-614, 1998.

[54] N. Hardy, N. S. Magner, J. Lavin, R. A. Cardenas, and M. JaraBertin, "Small consequences of a major agreement: the MILA case," Academia Revista Latinoamericana de Administración, vol. 31, no. 3, pp. 486-518, 2018.

[55] S. J. Hussain Shahzad, E. Bouri, J. Arreola-Hernandez, D. Roubaud, and S. Bekiros, "Spillover across Eurozone credit market sectors and determinants," Applied Economics, vol. 51, no. 59, pp. 6333-6349, 2019.

[56] J.-P. Onnela, A. Chakraborti, K. Kaski, and J. Kertész, "Dynamic asset trees and black monday," Physica A: Statistical Mechanics and Its Applications, vol. 324, no. 1-2, pp. 247-252, 2003.

[57] T. Araújo and F. Louçã, "The geometry of crashes. A measure of the dynamics of stock market crises," Quantitative Finance, vol. 7, no. 1, pp. 63-74, 2007.

[58] M. Tumminello, T. Aste, T. Di Matteo, and R. N. Mantegna, "A tool for filtering information in complex systems," Proceedings of the National Academy of Sciences, vol. 102, no. 30, pp. 10421-10426, 2005.

[59] M. Tumminello, F. Lillo, and R. N. Mantegna, "Correlation, hierarchies, and networks in financial markets," Journal of Economic Behavior \& Organization, vol. 75, no. 1, pp. 40-58, 2010.

[60] J. Birch, A. A. Pantelous, and K. Soramäki, "Analysis of correlation based networks representing DAX 30 stock price 
returns," Computational Economics, vol. 47, no. 4, pp. 501-525, 2016.

[61] W. K. Newey and K. D. West, "A simple, positive semidefinite, heteroskedasticity and autocorrelation consistent covariance matrix," Econometrica, vol. 55, no. 3, pp. 703-708, 1987.

[62] W. K. Newey and K. D. West, "Automatic lag selection in covariance matrix estimation," The Review of Economic Studies, vol. 61, no. 4, pp. 631-653, 1994.

[63] T. E. Clark and M. W. McCracken, "Tests of equal forecast accuracy and encompassing for nested models," Journal of Econometrics, vol. 105, no. 1, pp. 85-110, 2001.

[64] T. E. Clark and K. D. West, "Using out-of-sample mean squared prediction errors to test the martingale difference hypothesis," Journal of Econometrics, vol. 135, no. 1-2, pp. 155-186, 2006.

[65] T. E. Clark and K. D. West, "Approximately normal tests for equal predictive accuracy in nested models," Journal of Econometrics, vol. 138, no. 1, pp. 291-311, 2007.

[66] R. Giacomini and H. White, "Tests of conditional predictive ability," Econometrica, vol. 74, no. 6, pp. 1545-1578, 2006.

[67] K. D. West, "Forecast evaluation," Handbook of Economic Forecasting, vol. 1, pp. 99-134, Elsevier, Amsterdam, Netherlands, 2006.

[68] T. Clark and M. McCracken, "Advances in forecast evaluation," Handbook of Economic Forecasting, vol. 2, pp. 11071201, Elsevier, Amsterdam, Netherlands, 2013.

[69] B. Rossi, "Advances in forecasting under instability," Handbook of Economic Forecasting, vol. 2, pp. 1203-1324, Elsevier, Amsterdam, Netherlands, 2013.

[70] A. Sensoy and B. M. Tabak, "Dynamic spanning trees in stock market networks: the case of Asia-Pacific," Physica A: Statistical Mechanics and Its Applications, vol. 414, pp. 387-402, 2014.

[71] S. M. Guerra, T. C. Silva, B. M. Tabak, R. A. de Souza Penaloza, and R. C. de Castro Miranda, "Systemic risk measures," Physica A: Statistical Mechanics and Its Applications, vol. 442, pp. 329-342, 2016.

[72] B. Kadirvelu, Y. Hayashi, and S. J. Nasuto, "Inferring structural connectivity using Ising couplings in models of neuronal networks," Scientific Reports, vol. 7, no. 1, pp. 1-12, 2017. 